\title{
A NOTE ON THE CONSISTENCY OF LIMITATION METHODS
}

B. CHOUDHARY AND S. K. MISHRA

1.

We denote by $w$ the space of all real or complex valued sequences. We consider matrix transformations $y_{i}=\sum_{j=0}^{\infty} a_{i, j} x_{j}$ (that is, $y=A x$, where $x, y \in$ $w$ ). We write $w_{A}=\{x: A x \in w\}$. Let $m, b v, c, c^{0}$ be respectively the spaces of bounded, bounded variation, convergent and null sequences; $\ell=\{x$ : $\left.\sum_{n=0}^{\infty}\left|x_{n}\right|<\infty\right\} ; b v^{0}=b v \cap c^{0}$. We shall make use of the special sequences $e^{k}=(0,0,0, \cdots, 1,0,0,0, \cdots), e=(1,1,1, \cdots)$. Denote $\Delta=\left\{e^{k}\right\}_{k>0}$ and $\Delta^{+}=$ $\Delta+\{e\}$. We now define a sequence space of different type, namely

$$
b_{A}=\left\{x \in w_{A}:\left|\sum_{k=m}^{\infty} a_{n k} x_{k}\right| \leq K(A, x) \quad(m, n=0,1,2, \cdots)\right\} .
$$

If $x \in c$ its limit is denoted by $\lim x$, and if $x \in c_{A}$ we denote $\lim (A x)=$ $\lim _{A} x$. Denote the set of all $e$ 's by

$$
\phi=\left\{e, e^{0}, e^{1}, e^{2}, \cdots\right\}
$$

Then $e \in c_{A}, e^{k} \in c_{A}$ are respectively equivalent to the requirements that row sums of $A$ exist and tend to a limit, or that the $k$-th column-limit of $A$ exists. We denote $\chi_{A}=\lim _{A} e-\sum_{k} \lim _{A} e^{k}$ whenever this exists and is finite, and 
$\|A\|=\sup _{n} \sum_{k}\left|a_{n k}\right|$, finite or not. Note that $\ell_{A} \subseteq c_{A}^{0} \subseteq c_{A} \subseteq m_{A} \subseteq w_{A}$ and $\ell_{A} \subseteq b v_{A} \subseteq c_{A}$.

We say that $A$ defines a section bounded matrix transformation (introduced by Wilansky and Zeller [7]), when it belongs to the set

$$
\mathcal{A}=\left\{A: c_{A} \subseteq b_{A}\right\}
$$

Thus matrices in $\mathcal{A}$ have the property

$$
\mathcal{A}: \forall x \in c_{A}, \sup _{m, n}\left|\sum_{k=m}^{\infty} a_{n k} x_{k}\right|=\mathbb{K}(A, x)<\infty .
$$

We define

$$
\mathcal{A}^{0}=\left\{A: c_{A}^{0} \subseteq b_{A}\right\} .
$$

A real matrix $H=\left(h_{n k}\right)$ is called diagonal positive when

$$
h_{n k}<0(0 \leq k<n), h_{n n}>0, h_{n k}=0(k>n) ; n, k=0,1,2, \cdots,
$$

2.

In [2] the authors have obtained necessary and sufficient conditions for the consistency of two limitation methods for Nörlund summable sequences. The object of this note is to extend the results obtained in [2] and demonstrate that by using functional analysis technique the proof becomes easier.

In order to state our theorem we need some definitions.

Let $p=\left(p_{n}\right)$ and $q=\left(q_{n}\right)$ be sequences of real or complex numbers such that $q_{n} \neq 0$ for $n \geq 0$ and $q_{n}=0, p_{n}=0$, for $n<0$. Let

$$
r_{n}=\sum_{k=0}^{n} p_{n-k} q_{k} .
$$

We assume that, for all $n, r_{n} \neq 0$. Let $\sum_{n=0}^{\infty} a_{n}$ be an infinite series with $\left(s_{n}\right)$ as the sequence of its partial sums. Let $(N, p, q)$ denotes the generalized Nörlund 
method in which the sequence $\left(s_{n}\right)$ is transformed into $\tau_{n}$ given by

$$
\tau_{n}=\frac{1}{r_{n}} \sum_{k=0}^{n} p_{n-k} q_{k} s_{k}
$$

If $\tau_{n} \rightarrow s$ (finite) as $n \rightarrow \infty$, then the series $\sum_{n=0}^{\infty} a_{n}$ (or the sequence $\left(s_{n}\right)$ ) is said to be summable by the generalized Nörlund method $(N, p, q)$ to $s$. We denote it by

$$
\sum_{n=0}^{\infty} a_{n}=s(N, p, q) \quad \text { or } \quad s_{n} \rightarrow s(N, p, q) .
$$

The necessary and sufficient conditions for the regularity of $(N, p, q)$ are

$$
\sum_{v=0}^{n}\left|p_{n-v} q_{v}\right|=0\left[\left|r_{n}\right|\right] \quad(n \geq 0)
$$

and

$$
p_{n-v}=0\left[\left|r_{n}\right|\right] \text { as } n \rightarrow \infty \quad(v \text { fixed }) .
$$

This follows from Toeplitz's theorem (Hardy [1], Theorem 2). The method $(N, p, q)$ reduces to the Nörlund method $(N, p)$ when $q_{n}=1$ for all $n$ (Hardy [1], p.64) and to the Riesz method $(\bar{N}, q)$ when $p_{n}=1$ for all $n$ (Hardy [1], p.57).

Given any sequence $\left(p_{n}\right)$, we write

$$
p(z)=\sum_{n=0}^{\infty} p_{n} z^{n}, \quad p_{-m}>0 \quad(m>0)
$$

and

$$
[p(z)]^{-1}=\sum_{n=0}^{\infty} \gamma_{n} z^{n}
$$

whenever the series on the right converges.

It is reasonably familiar that if we define

$$
z_{n}=\sum_{k=0}^{n} p_{n-k} y_{k}
$$

then 


$$
y_{n}=\sum_{k=0}^{n} \gamma_{n-k} z_{k}
$$

Applying this with $y_{k}=s_{k} q_{k}, z_{k}=r_{k} \tau_{k}$ we find that

$$
s_{n}=\frac{1}{q_{n}} \sum_{k=0}^{n} \gamma_{n-k} \tau_{k} r_{k}
$$

As usual we say that the sequence $\left(p_{n}\right) \in \mathcal{M}$, if $p_{0}=1$,

$$
p_{n}>0, \frac{p_{n+1}}{p_{n}} \leq \frac{p_{n+2}}{p_{n+1}} \leq 1 \quad(n=0,1, \cdots)
$$

Let $A$ and $B$ be two infinite matrices.

Let

$$
u_{m}=\sum_{n=0}^{\infty} a_{m n} s_{n}, \quad v_{m}=\sum_{n=0}^{\infty} b_{m n} s_{n} .
$$

We say that the $A, B$ transforms are equiconvergent if

$$
\sum_{n=0}^{\infty}\left(a_{m n}-b_{m n}\right) s_{n} \quad \text { converges for all } m
$$

and its sum tends to 0 as $m \rightarrow \infty$. We shall prove the following theorem.

Theorem 1. Let $(N, p)$ be a Nörlund method where $p_{n} \in \mathcal{M}$ and let $\left(q_{n}\right)$ be a sequence where each $q_{n} \geq 1$. Then the necessary and sufficient conditions that $A, B$ transforms are equiconvergent for all $(N, p, q)$ summable sequences are

(i) $\lim _{m \rightarrow \infty} d_{m n}=0$ for every fixed $n$;

(ii) $\lim _{m \rightarrow \infty} \sum_{n=0}^{\infty} d_{m n}=0$

(iii) $\sup _{m} \sum_{k=0}^{\infty} r_{k}\left|\sum_{n=k}^{\infty} \frac{d_{m n}}{q_{n}} \gamma_{n-k}\right| \leq M$

where $d_{m n}=a_{m n}-b_{m n}$.

In order to prove the theorem we need some known results. We shall see that by using functional anaylysis technique the proof of Theorem 1 becomes easier. 
Lemma 1. If $p \in \mathcal{M}$ and if $q_{n} \geq \delta>0, r_{n}=\sum_{k=0}^{n} p_{n-k} q_{k}$, then $H=(N, p, q)$ is non-negative, normal, regular, and has the normal inverse

$$
H^{-1}=\left(h_{n k}^{-1}\right), h_{n k}^{-1}=r_{k} \bar{p}_{n-k} / q_{n} \quad(0 \leq k<n),
$$

where $\bar{p}_{0}=1 / p_{0}, \bar{p}_{k} \leq 0(k \geq 1)$; also $\sum_{k=0}^{n} h_{n k}^{-1}=1$. It follows that $H$ satisfies $h_{n k}^{-1} \leq 0(0 \leq k \leq n), \sum_{k=0}^{n} h_{n k}^{-1} \geq 0$, and hence $H$ has the section bounded property.

This result is known and given in ([7] Kriterium 1, p. 260).

Theorem $\mathbb{A}$. Let $H$ be normal, section bounded, and coregular. In order that $c_{H} \subseteq c_{D}$ it is necessary and sufficient that

(i) $X_{D}$ exists;

(ii) $\left\|D H^{-1}\right\|<\infty$

This theorem follows from a Theorem ([4], Theorem 3, p. 263).

Suppose that $\triangle=\left\{e^{k}\right\} \subseteq c_{H}^{0}$. This means that $c_{H}^{0}$ is an $A K$-space $(\triangle$ is a Schauder basis for $\left.c_{H}^{0}\right)$.

Theorem B. Let $H$ be normal, section-bounded, and $\triangle \subseteq c_{H}^{0}$. Then

$$
c_{H}^{0} \subseteq c_{D}^{0} \Longleftrightarrow\left\{\begin{array}{l}
\triangle \subseteq c_{D}^{0} \\
T=D H^{-1} \text { satisfies } D=T H,\|T\|<\infty
\end{array}\right.
$$

The proof of this theorem is similar to the proof given in ([4], Theorem 4). We shall now extend Theorem B. In order to extend Theorem $B$ we need a very simple lemma.

Lemma 2. If there is a sequence $\delta$ such that $H \delta=e$, then

$$
c_{H} \subseteq c_{D}^{0} \Longleftrightarrow \delta \in c_{D}^{0} \text { and } c_{H}^{0} \subseteq c_{D}^{0} .
$$

In the particular application $H=(N, p, q)$, we have $\sum_{k=0}^{n} h_{n k}=1$ for every $n$, or that $H e=e($ i.e. $\delta=e)$. So if we write $\Delta^{+}=\triangle \cup\{e\}=\left\{e^{k}\right\} \cup\{e\}$, Theorem $B$ and Lemma 2 yield: 
Theorem C. Let $H=(N, p, q)$ where $p \in \mathcal{M}$ and $q_{n} \geq \delta>0$. Then

$$
c_{H} \subseteq c_{D}^{0} \Leftrightarrow\left\{\begin{array}{l}
\triangle^{+} \subseteq c_{D}^{0} \\
T=D H^{-1} \text { satisfies } D=T H,\|T\|<\infty
\end{array}\right.
$$

Remark. More serious is the elimination of the criterion " $T=D H^{-1}$ satisfies $D=T H$ " in Theorem C.

We shall now show that for the matrix $H=(N, p, q)$, this is true.

In order to see this we need the following results:

Lemma 3. Let $H$ be normal and have the inverse property. Let $u$ be a positive sequence such that $u H^{-1}$ exists and is non-negative. If $d$ is a sequence such that

$$
\begin{aligned}
d_{k} & =o\left(u_{k}\right) \text { then } d H^{-1} \text { and }\left(d H^{-1}\right) H \text { exist, and } \\
d & =\left(d H^{-1}\right) H
\end{aligned}
$$

Proof. Note that $H$ is non-negative. Since $H^{-1}$ is diagonal positive and $u H^{-1}$ exists, it follows that

$$
\sum_{n=0}^{\infty} u_{n}\left|h_{n k}^{-1}\right|<\infty, \quad \text { and }
$$

$d_{n}=o\left(u_{n}\right)$. This shows that $d H^{-1}$ exists.

Choose any fixed $r \geq 0$; then, for any $N \geq r$

$$
\begin{aligned}
d_{r} & =\sum_{n=r}^{N} d_{n} \delta_{n r} \\
& =\sum_{n=r}^{N} d_{n} \sum_{k=r}^{n} h_{n k}^{-1} h_{k r} \\
& =\left(\sum_{k=r}^{N} \sum_{n=k}^{\infty}-\sum_{n=r}^{N} \sum_{n=N+1}^{\infty}\right) d_{n} h_{n k}^{-1} h_{n r} \\
& \left.=S_{1}-S_{2} \text { (since } d H^{-1} \text { exists }\right) .
\end{aligned}
$$


Write

$$
\tau_{N}=\sup _{n>N+1}\left|\frac{d_{n}}{u_{n}}\right|=o(1) \text { as } N \rightarrow \infty, \quad \text { since } \frac{d_{n}}{u_{n}}=o(1)
$$

Then

$$
\begin{aligned}
\left|S_{2}\right| & \leq \sum_{k=r}^{N} h_{k r} \sum_{n=N+1}^{\infty}\left|d_{n} h_{n k}^{-1}\right| \\
& \leq \tau_{N} \sum_{k=r}^{N} h_{k r}\left(-\sum_{n=N+1}^{\infty} u_{n} h_{n k}^{-1}\right)
\end{aligned}
$$

since $h_{n k}^{-1} \leq 0$ for $n>k$,

$$
\leq \tau_{N} \sum_{k=r}^{N} h_{k r} \sum_{n=k}^{N} u_{n} h_{n k}^{-1},
$$

since $\left(\sum_{n=k}^{N}+\sum_{n=N+1}^{\infty}\right) u_{n} h_{n k}^{-1} \geq 0$

$$
\begin{aligned}
& =\tau_{N} \sum_{n=r}^{N} u_{n} \sum_{k=r}^{n} h_{n k}^{-1} h_{k r} \\
& =\tau_{N} \sum_{n=r}^{N} u_{n} \delta_{n r}=\tau_{N} u_{r} \rightarrow 0 \text { as } N \rightarrow \infty
\end{aligned}
$$

It follows that $d_{r}=\lim _{N \rightarrow \infty} S_{1}=\left[\left(d H^{-1}\right) H\right]_{r}$ for $r=0,1,2, \cdots$

Lemma 4. Let $H$ be normal and have the inverse property. Let $u$ be a positive sequence such that

$$
u_{k+1} h_{n k} \leq u_{k} h_{n, k+1} \quad(0 \leq k<n=1,2, \cdots) .
$$

Then $u H^{-1}$ exists and is non-negative.

Proof. Write $T=\left(t_{n k}\right), t_{n k}=1(0 \leq k \leq n), t_{n k}=0(k>n)$; its inverse is

$$
T^{-1}=\left(t_{n k}^{-1}\right), t_{n n}^{-1}=1, t_{n n-1}^{-1}=-1, t_{n k}^{-1}=0 \text { otherwise. }
$$


Let

$$
\begin{aligned}
g_{n k} & =\frac{h_{n k}}{u_{n}} ; \text { then } g_{n k}^{-1}=u_{n} g_{n k}^{-1} \text { and, by hypothesis, } \\
\left(G T^{-1}\right)_{n k} & =g_{n k}-g_{n, k+1} \leq 0 \text { for } 0 \leq k<n=1,2,3, \cdots
\end{aligned}
$$

Hence, $\left(G T^{-1}\right)^{-1}=T G^{-1}$ is non-negative. That is,

$$
0 \leq\left(T G^{-1}\right)_{N K}=\sum_{n=k}^{N} u_{n} h_{n k}^{-1}=u_{k} h_{n k}^{-1}-\sum_{n=k+1}^{N} u_{n}\left|h_{n k}^{-1}\right|,
$$

and so $\sum_{n=k}^{\infty} u_{n} h_{n k}^{-1}$ converges and is non-negative, for each $k$.

In the case $H=(N, p, q)$ we have

$$
h_{n k}=\frac{p_{n-k} q_{k}}{r_{n}} \quad\left(r_{n}=\sum_{k=0}^{n} p_{n-k} q_{k}\right)
$$

Since $p \in \mathcal{M}$, it follows that

$$
\frac{h_{n k}}{q_{k}} \leq \frac{h_{n, k+1}}{q_{k+1}}
$$

Hence, $u_{k}=q_{k}$ in the last two lemmas. This gives:

If $d_{k}=o\left(q_{k}\right)$, then $t=d H^{-1}$ exists and satisfies $d=t H$ where $H=(N, p, q)$. Moreover, since $q_{k} \geq \delta>0$. It follows that

$$
d_{k}=o(1) \Rightarrow d_{k}=o\left(q_{k}\right)
$$

Replacing $d_{k}$ by $d_{n k}$ we have:

If $\lim _{k} d_{n k}=0$ for each $n$, then $T=D H^{-1}$ exists and satisfies $D=T H$. Finally, the condition $e \in c_{D}^{o}$ (included in the condition $\Delta^{+} \subseteq c_{D}^{o}$ of Theorem C) says that

$$
\begin{aligned}
& \lim _{n} \sum_{k=0}^{\infty} d_{n, k}=0, \quad \text { and in particular this implies that } \\
& \lim _{k} d_{n k}=0 \text { for each } n .
\end{aligned}
$$

Thus Theorem $\mathrm{C}$ reduces to the form 
Theorem $\mathrm{C}^{\prime}$ Let $H=(N, p, q)$, where $p \in \mathcal{M}$ and $q_{n} \geq \delta>0$. Then

$$
c_{H} \subseteq c_{D}^{o} \Longleftrightarrow \triangle^{+} \subseteq c_{D}^{o} \text { and }\left\|D H^{-1}\right\|<\infty
$$

The conditions on the right of Theorem $C^{\prime}$ are:

(i) $\lim _{m} d_{m n}=0$ for each $n\left(e^{n} \in c_{D}^{o}\right)$.

(ii) $\lim _{m} \sum_{n=0}^{\infty} d_{m n}=0\left(e \in c_{D}^{o}\right)$

(iii) $\sup _{m} \sum_{k=0}^{\infty} r_{k}\left|\sum_{n=k}^{\infty} \frac{d_{m n}}{q_{n}} \gamma_{n-k}\right|<M\left(\left\|D H^{-1}\right\|<\infty\right)$.

Thus the proof of Theorem 1 is complete.

\section{References}

[1] G. H. Hardy, "Divergent Series", Oxford University Press, 1949.

[2] N. Kishore and U. Mishra, "On the consistency of limitation methods for $\left(N, p_{n}\right)$ summable sequences", Int. J. Math. and Math. Sc., Vol. 4, No. 1, (1981).

[3] A. Jakimovski and D. C. Russell, "On beta-duals of Matrix fields", Commentationes Mathematicae II (1979), 159-171.

[4] D. C. Russell, "Inclusion theorems for section-bounded matrix transformations", Math. Zeit. 113 (1970), 255-265.

[5] D. C. Russell, "Note on Inclusion Theorems for infinite matrices", J. London Math. Soc., 33 (1958), 50-62.

[6] A. Wilansky, "Functional Analysis", Blaisdell, New York, 1964.

[7] A. Wilansky and K. Zeller, "Abschnittsbeschrünkte Matrixtransformationen Starks Limitierbarkeit", Math. Zeit, 64 (1956), 258-269.

[8] K. Zeller, "Allgemeine Eigenschaften von Limitierunge-Verfahren", Math. Zeit., (1953), 463-487.

[9] K. Zeller, "Abschnittsa bschatzungen bei Matrixtransformationen", ibidem 80 (1963), 355-357.

Department of Mathematics, Indian Institute of Technology, Hauz Khas, New Delhi-110016, India. 